\title{
CIRCULARIZABLE DOMAINS ON RIEMANN SURFACES
}

\author{
Makoto Masumoto and Masakazu Shiba \\ To the memory of Professor Nobuyuki Suita
}

\section{Introduction}

Let $R$ be a Riemann surface and $\Gamma$ a properly discontinuous group of conformal automorphisms of $R$. For the sake of simplicity we assume that every nontrivial element of $\Gamma$ has no fixed points in $R$. Suppose that $R / \Gamma$ is conformally equivalent to neither the plane $\mathbf{C}$ nor the sphere $\hat{\mathbf{C}}$. For $p \in R$ let $\mathscr{D}_{p}^{\Gamma}(R)$ denote the class of simply connected domains $D$ on $R$ containing $p$ such that $\gamma(D) \cap D=\emptyset$ for any $\gamma \in \Gamma \backslash\left\{\operatorname{id}_{R}\right\}$. Then every $D \in \mathscr{D}_{p}^{\Gamma}(R)$ carries a unique complete conformal metric $d s_{D}$ with curvature -1 . In our previous work [4] we considered the function $D \mapsto\left(d s_{R} / d s_{D}\right)(p)$ on $\mathscr{D}_{p}^{\Gamma}(R)$, where $d s_{R}$ denotes a complete conformal metric on $R$ with constant curvature, and proved that $\mathscr{D}_{p}^{\Gamma}(R)$ contains a unique element that maximizes the function. Such an element is called a hyperbolically maximal domain for $\Gamma$.

Hyperbolically maximal domains possess distinguished properties. For example, they are locally finite fundamental domains for $\Gamma$. Each hyperbolically maximal domain $D$ is associated with a $\Gamma$-invariant meromorphic quadratic differential $\varphi$ such that $D \backslash\{p\}$ is swept out by closed horizontal trajectories of $\varphi$; the point $p$ is a double pole of $\varphi$.

This property of hyperbolically maximal domains leads us to the study of domains dominated by quadratic differentials. Specifically, we call a domain $D$ on $R$ a $\Gamma$-circularizable domain if there is a $\Gamma$-invariant meromorphic quadratic differential $\varphi$ with poles in $\bigcup_{\gamma \in \Gamma} \gamma(D)$ such that every noncritical point in $D$ lies on a closed horizontal trajectory of $\varphi$ that stays entirely in $D$. Circularizable domains are subject to fairly strong topological restrictions. They turn out to be simply connected domains whose boundaries are locally finite branched polygons.

Several analytic and geometric properties of hyperbolically maximal domains given in [4] are shared with circularizable domains. In fact, hyperbolically maximal domains for $\Gamma$ are $\Gamma$-circularizable and those properties come from circularizability. Circularizable domains thus deserve to be studied in detail. We begin with the definition of a circularizable domain. In $\$ 3$ we show that a circularizable domain $D$ satisfies $\gamma(D) \cap D=\emptyset$ for $\gamma \in \Gamma \backslash\left\{\operatorname{id}_{R}\right\}$ provided $D$

Received February 18, 2004; revised August 11, 2004. 
contains no fixed points of nontrivial elements of $\Gamma$. In $\S 4$ we investigate the boundaries of circularizable domains. In the final section we give a necessary and sufficient condition for a $\Gamma$-circularizable domain to be hyperbolically maximal for $\Gamma$.

The authors are grateful for the referee's careful reading.

\section{Basic properties}

Let $R$ be any Riemann surface and $\varphi$ a meromorphic quadratic differential on $R$. We denote by $\operatorname{ord}_{q} \varphi$ the algebraic order of $\varphi$ at $q \in R$. For a subset $E$ of $R$ we mean by $P_{\varphi}(E)$ the set of poles of $\varphi$ in $E$. In other words $P_{\varphi}(E)$ is the set of points $q \in E$ for which $\operatorname{ord}_{q} \varphi \leqq-1$. Its cardinality is denoted by ${ }^{\#} P_{\varphi}(E)$.

Definition 2.1. We say that a domain $D$ on $R$ is circularizable if there is a meromorphic quadratic differential $\varphi$ on $R$ such that

(i) $P_{\varphi}(D) \neq \emptyset$ and

(ii) the (maximal) horizontal trajectory passing through any noncritical point of $\varphi$ in $D$ is closed and stays entirely in $D$.

The differential $\varphi$ is called a circularizer of $D$. The pair $(D, \varphi)$ is called a circularized domain.

Remark. Assume that $R$ is a subdomain of another Riemann surface $R^{\prime}$. If a domain $D$ on $R$ is a circularizable domain on $R^{\prime}$, then, trivially, $D$ is a circularizable domain on $R$. However, the converse is not necessarily true because circularizers of $D$ on $R$ may not be extended meromorphically on $R^{\prime}$.

Example 2.1. Let $\mathbf{D}$ be the unit disk in the complex plane $\mathbf{C}$, and set $\hat{\mathbf{C}}=\mathbf{C} \cup\{\infty\}$. For $a \in \mathbf{D}$ set $\varphi_{a}=-d z^{2} /(z-a)^{2}(1-\bar{a} z)^{2}$. Then $\left(\hat{\mathbf{C}}, \varphi_{a}\right),\left(\hat{\mathbf{C}}_{a}, \varphi_{a}\right)$ and $\left(\mathbf{D}, \varphi_{a}\right)$ are circularized domains on $\hat{\mathbf{C}}$, where $\hat{\mathbf{C}}_{a}=\hat{\mathbf{C}} \backslash\{1 / \bar{a}\}$.

It should be noted that all the domains in Example 2.1 are simply connected. In fact we have the following proposition.

Proposition 2.1. A circularizable domain is simply connected.

Proof. Let $(D, \varphi)$ be a circularized domain on $R$. Take a noncritical point of $\varphi$ in $D$. By (ii) the horizontal trajectory $\alpha$ of $\varphi$ passing through the point is closed and stays entirely in $D$. Consider a subdomain $\Delta$ of $D$ which includes $\alpha$ and is swept out by closed horizontal trajectories of $\varphi$ freely homotopic in $\Delta$ to $\alpha$. We may assume that $\Delta$ is maximal with respect to the inclusion relation. Then $\Delta$ is either a torus or a ring domain (see Strebel $[5, \S 9]$ ). If $\Delta$ is a torus, then it coincides with $R$ by compactness, and hence we have $P_{\varphi}(R)=P_{\varphi}(\Delta)=\emptyset$, which contradicts (i). Thus $\Delta$ must be a ring domain.

We claim that the relative boundary $\beta:=\partial \Delta \cap D$ of $\Delta$ in $D$ is nonempty and discrete. To see that $\beta \neq \emptyset$ we have only to note that $\varphi$ is holomorphic 
everywhere on $\Delta$ and has at least one pole in $D$. If $\beta$ were not discrete, then it would contain a noncritical point $q$ of $\varphi$. The closed horizontal trajectory $\tau$ of $\varphi$ containing $q$ stays entirely in $\beta$, so that $\tau$ is a component of $\beta$. It is now easy to construct a ring domain $\Delta^{\prime}$ swept out by closed horizontal trajectories of $\varphi$ such that $\Delta \cup \tau \varsubsetneqq \Delta^{\prime} \subset D$, which contradicts the maximality of $\Delta$.

For any $p \in \beta$, by discreteness, there exists a connected neighborhood $U \subset D$ of $p$ such that $U \cap \beta=\{p\}$. The punctured neighborhood $U \backslash\{p\}$ is connected and is hence included in $\Delta$. This implies that $\Delta \cup \beta$ is open in $D$. Since it is also closed in $D$, it follows that $\Delta \cup \beta=D$. We have $\Delta=D \backslash \beta$, for $\Delta \cap \beta=\emptyset$. This shows that the connectivity of $D$ is smaller than that of $\Delta$. Since $\Delta$ is doubly connected, we conclude that $D$ is simply connected.

Remark. As is seen from the above proof, $\beta=D \backslash \Delta$. Since $P_{\varphi}(D) \subset D \backslash \Delta$, we know that $\beta \supset P_{\varphi}(D)$. If $\beta \backslash P_{\varphi}(D)$ were nonempty, we could take $q \in$ $\beta \backslash P_{\varphi}(D)$. Since $\varphi$ is holomorphic at $q$, there is a horizontal arc $\tau$ of $\varphi$ emanating from $q$. By the discreteness of $\beta$ we have $\tau \cap \Delta \neq \emptyset$. Let $\tau^{\prime}$ be the closed horizontal trajectory of $\varphi$ passing through a point of $\tau \cap \Delta$. Then $\tau \subset \tau^{\prime} \subset \Delta$. Since $q$ is an end point of $\tau$, it belongs to $\tau^{\prime}$ so that $q \in \Delta$. This contradicts $q \in \beta$. Thus $\beta=P_{\varphi}(D)$.

If Riemann surfaces $R_{1}$ and $R_{2}$ are conformally equivalent to each other, then we write $R_{1} \cong R_{2}$. The following corollary follows at once from Proposition 2.1 .

COROllary 2.1. Let $D$ be a circularizable domain on $R$.

(i) If $\partial D=\emptyset$, then $R=D \cong \hat{\mathbf{C}}$, $\mathbf{C}$ or $\mathbf{D}$.

(ii) If $\partial D$ contains an isolated boundary point, then $R \cong \hat{\mathbf{C}}$ and $D \cong \mathbf{C}$.

The following proposition concerns the behavior of circularizers on a corresponding circularizable domain.

Proposition 2.2. Let $(D, \varphi)$ be a circularized domain on $R$. Then

(i) $D \backslash P_{\varphi}(D)$ is swept out by closed horizontal trajectories of $\varphi$, which are of the same $\varphi$-length,

(ii) ${ }^{\#} P_{\varphi}(D)=1$ or 2 ,

(ii') ${ }^{\#} P_{\varphi}(D)=2 \Leftrightarrow D$ is compact $\Leftrightarrow D \cong \hat{\mathbf{C}}$, and

(iii) $\operatorname{ord}_{q} \varphi=-2$ for $q \in P_{\varphi}(D)$.

Proof. Let $\Delta$ and $\beta$ be as before. Since $D \backslash P_{\varphi}(D)=D \backslash \beta=\Delta$, we have the first half of (i). The rest of (i) follows from the general theory (see $[5, \S 9.3])$. Taking the connectivities of $D$ and $\Delta$ into account, we obtain (ii) and (ii'). For $q \in P_{\varphi}(D)$ there is a domain $G$ with $q \in G$ such that $\partial G$ is a closed horizontal trajectory of $\varphi$ in $\Delta$. We can apply Teichmüller's lemma (cf. [5, Theorem 14.1]) to verify assertion (iii). 
Remark. There exists a conformal mapping $w$ of $D$ into $\hat{\mathbf{C}}$ such that

$$
\varphi=-c \frac{d w^{2}}{w^{2}}
$$

for some positive constant $c$. The image $w(D)$ is either the sphere or the plane or a disk centered at the origin. The domain $D$ carries a complete conformal metric $d \sigma_{D}$ of constant curvature $\kappa$, where

$$
\begin{cases}\kappa>0 & \text { if } w(D) \text { is the sphere } \\ \kappa=0 & \text { if } w(D) \text { is the plane } \\ \kappa<0 & \text { if } w(D) \text { is a disk. }\end{cases}
$$

The closed horizontal trajectories of $\varphi$ in $D$ are concentric circles with respect to the metric $d \sigma_{D}$. Compare this fact with [3, Theorem 1].

Now assume that a group $\Gamma$ of conformal automorphisms of $R$ acts properly discontinuously on $R$. A $\Gamma$-circularizable domain on $R$ is, by definition, a circularizable domain whose circularizer $\varphi$ is $\Gamma$-invariant. We call $\varphi$ a $\Gamma$-circularizer of the domain, and the pair $(D, \varphi)$ a $\Gamma$-circularized domain.

If $(D, \varphi)$ is a $\Gamma$-circularized domain, then so is $(\gamma(D), \varphi)$ for any $\gamma \in \Gamma$. If, in addition, $D$ is noncompact, then $P_{\varphi}(D)=\{p\}$ and $P_{\varphi}(\gamma(D))=\{\gamma(p)\}$ for some $p \in D$ by Proposition 2.2 (ii) and (ii').

Important examples of circularizable domains are hyperbolically maximal domains introduced in [4]. We first recall the definition. Let $\mathscr{D}^{\Gamma}(R)$ be the class of simply connected domains $D$ on $R$ such that $\gamma(D) \cap D=\emptyset$ for all $\gamma \in \Gamma \backslash\left\{\mathrm{id}_{R}\right\}$. Denote by $R^{*}$ the set of points of $R$ which are fixed by no nontrivial elements of $\Gamma$. For $p \in R^{*}$ let $\mathscr{D}_{p}^{\Gamma}(R)$ be the set of $D \in \mathscr{D}^{\Gamma}(R)$ with $p \in D$. When $\Gamma=\left\{\operatorname{id}_{R}\right\}$, we abbreviate $\mathscr{D}^{\Gamma}(R)$ and $\mathscr{D}_{p}^{\Gamma}(R)$ to $\mathscr{D}(R)$ and $\mathscr{D}_{p}(R)$, respectively. If $R^{*} / \Gamma \nsubseteq \mathbf{C}, \hat{\mathbf{C}}$, then any $D \in \mathscr{D}_{p}^{\Gamma}(R)$ is conformally equivalent to D so that it carries a (unique) complete conformal metric $d s_{D}$ with constant curvature -1 . For $D_{1}, D_{2} \in \mathscr{D}_{p}^{\Gamma}(R)$ we write $D_{1} \preceq D_{p}$ if $\left(d s_{D_{2}} / d s_{D_{1}}\right)(p) \leqq 1$. Then $\left(\mathscr{D}_{p}^{\Gamma}(R), \preceq\right)$ is a quasi-ordered class, which contains a unique maximum ([4, Theorem $\left.3.1^{\prime}\right]$ ); A simply connected domain $D$ on $R$ is called hyperbolically maximal for $\Gamma$ if it is maximal in $\left(\mathscr{D}_{p}^{\Gamma}(R), \underset{p}{\preceq}\right)$ for some $p \in D \cap R^{*}$.

The next proposition is an immediate consequence of [4, Proposition 3.1].

Proposition 2.3. A hyperbolically maximal domain for $\Gamma$ is $\Gamma$-circularizable.

Compact $\Gamma$-circularizable domains appear only in special cases. To show this, we need the following lemma.

LEMma 2.1. Assume that $R \cong \hat{\mathbf{C}}$. If there is a $\Gamma$-invariant meromorphic quadratic differential $\varphi$ with ${ }^{\#} P_{\varphi}(R)=2$, then $\Gamma$ is cyclic or dihedral. 
Proof. Let $f: R \rightarrow \hat{\mathbf{C}}$ be conformal and set $G=f \circ \Gamma \circ f^{-1}$ and $Q=$ $f\left(P_{\varphi}(R)\right)$. Then $Q$ is $G$-invariant with ${ }^{\sharp} Q=2$. Since $G$ acts properly discontinuously on $\hat{\mathbf{C}}$, it is a finite group. By Jones-Singerman [2, Lemmas 2.13.3 and 2.13.4] it is cyclic or dihedral, and so is $\Gamma$.

Proposition 2.4. If there exists a compact $\Gamma$-circularizable domain on $R$, then $R$ is conformally equivalent to $\hat{\mathbf{C}}$ and $\Gamma$ is either cyclic or dihedral.

Proof. Let $D$ be a compact $\Gamma$-circularizable domain on $R$. Since $D$ is compact, we have $D=R$. On the other hand, $D \cong \hat{\mathbf{C}}$ by Proposition $2.2\left(\mathrm{ii}^{\prime}\right)$. Hence $R \cong \hat{\mathbf{C}}$. Another part of Proposition 2.2 (ii') together with Lemma 2.1 implies that $\Gamma$ is cyclic or dihedral.

Remark. The converse of Proposition 2.4 is also true. This will be verified at the end of the final section.

Proposition 2.5. Let $D$ be a $\Gamma$-circularizable domain on $R$. If the boundary $\partial D$ in $R$ is empty or contains an isolated point, then $\Gamma$ is either cyclic or dihedral.

The proof will be given after Proposition 3.1 is established.

\section{Behavior of $\Gamma$-circularizable domains under the action of $\Gamma$}

We next study the behavior of circularizable domains under the action of the discontinuous group. For $E \subset R$ we denote by $\Gamma_{E}$ the stabilizer in $\Gamma$ of $E$. When $E=\{p\}$, we abbreviate $\Gamma_{\{p\}}$ to $\Gamma_{p}$.

Lemma 3.1. If $(D, \varphi)$ is a $\Gamma$-circularized domain, then $\Gamma_{p} \subset \Gamma_{D}$ for any $p \in P_{\varphi}(D)$.

Proof. Let $p \in P_{\varphi}(D)$. If $D$ is compact, then $D=R$ so that $\Gamma_{D}=\Gamma \supset \Gamma_{p}$. Otherwise, $\gamma(D) \backslash\{\gamma(p)\}$ is a ring domain for any $\gamma \in \Gamma$. It is swept out by closed horizontal trajectories of $\gamma^{*} \varphi=\varphi$, where $\gamma^{*} \varphi$ denotes the pull-back of $\varphi$ via $\gamma$. It follows that for any $\gamma \in \Gamma_{p}$ either $D \subset \gamma(D)$ or $D \supset \gamma(D)$. By replacing $\gamma$ with $\gamma^{-1}$ if necessary, we may assume that $D \supset \gamma(D)$. Since $\Gamma$ acts properly discontinuously on $R$, the stabilizer $\Gamma_{p}$ is finite so that $\gamma^{k}=\mathrm{id}_{R}$ for some positive integer $k$. Therefore, we obtain

$$
D \supset \gamma(D) \supset \gamma^{2}(D) \supset \cdots \supset \gamma^{k}(D)=D
$$

and hence $\gamma(D)$ is identical with $D$, as desired.

Proposition 3.1. For any noncompact $\Gamma$-circularizable domain $D$ there exists $p \in D$ such that $\Gamma_{p}=\Gamma_{D}$. 
Proof. Let $\varphi$ be a $\Gamma$-circularizer of $D$. Since $D$ is noncompact, ${ }^{\#} P_{\varphi}(D)=1$ by Proposition 2.2 (ii) and (ii'), so that $\varphi$ has a single double pole $p$ in $D$. By Lemma 3.1 we have $\Gamma_{p} \subset \Gamma_{D}$. To show the converse inclusion relation recall that $P_{\varphi}(\gamma(D))=\{\gamma(p)\}$ holds for any $\gamma \in \Gamma$. If $\gamma \in \Gamma_{D}$, then $\{\gamma(p)\}=P_{\varphi}(\gamma(D))=$ $P_{\varphi}(D)=\{p\}$. This proves that $\Gamma_{p} \supset \Gamma_{D}$.

Remark. Let $D$ and $\varphi$ be as in Lemma 3.1. In the proof of Proposition 3.1 we have actually shown that if $D$ is noncompact, then $\Gamma_{p}=\Gamma_{D}$ for the pole $p$ of $\varphi$ in $D$.

Proof of Proposition 2.5. Assume first that $\partial D=\emptyset$. It follows from Corollary 2.1 (i) that $R$ is conformally equivalent to $\hat{\mathbf{C}}$ or $\mathbf{C}$ or $\mathbf{D}$. If $R \cong \hat{\mathbf{C}}$, then $D$ is compact so that $\Gamma$ is cyclic or dihedral by Proposition 2.4. Otherwise, we have $\Gamma=\Gamma_{D}=\Gamma_{p}$ for some $p \in D$ by Proposition 3.1. Since $\Gamma$ acts properly discontinuously on $R$, we know that $\Gamma=\Gamma_{p}$ is cyclic.

Next, if $\partial D$ contains an isolated point, then by Corollary 2.1 (ii) we know that $R \cong \hat{\mathbf{C}}$ and $D \cong \mathbf{C}$. In particular, $R \backslash D$ is a singleton, say $\{p\}$. Then $p$ is a critical point of a circularizer of $D$, for, otherwise, the horizontal trajectory of $\varphi$ passing through $p$ would meet $D$ without staying entirely in $D$. It follows that $(R, \varphi)$ is a circularized domain, and the assertion follows from Proposition 2.4 since $R$ is compact and $\Gamma$-circularizable.

Proposition 3.2. Suppose that one of the following conditions is satisfied:

(a) $R \not \hat{\mathbf{C}}$.

(b) $\Gamma$ is neither cyclic nor dihedral.

Let $D$ be a $\Gamma$-circularizable domain on $R$. Then for any $\gamma \in \Gamma$, either $\gamma(D)=D$ or $\gamma(D) \cap D=\emptyset$ holds.

Proof. Let $\varphi$ be a $\Gamma$-circularizer of $D$. It has a single double pole $p$ in $D$ since $D$ is noncompact by Proposition 2.4.

Suppose that $\gamma(D) \cap D \neq \emptyset$. By Lemma 3.1 we have only to show that $\gamma \in \Gamma_{p}$. If $\gamma(p)$ were different from $p$, then $P_{\varphi}(\gamma(D) \cap D)=P_{\varphi}(\gamma(D)) \cap P_{\varphi}(D)=$ $\{\gamma(p)\} \cap\{p\}=\emptyset$. By Proposition 2.2 (i) every point in $\gamma(D) \cap D$ lies on a closed horizontal trajectory of $\varphi$ staying entirely in $\gamma(D) \cap D$. By the same argument as in the proof of Proposition 2.1 we deduce that $\gamma(D) \cap D$ is a ring domain swept out by closed horizontal trajectories of $\varphi$. Since $p \in D \backslash \gamma(D)$ and $\gamma(p) \in \gamma(D) \backslash D$, the domain $\gamma(D) \cup D$ is topologically a sphere. Therefore $R \cong \hat{\mathbf{C}}$, which contradicts (a). Moreover, since ${ }^{\#} P_{\varphi}(R)=2$, it follows from Lemma 2.1 that $\Gamma$ is cyclic or dihedral, which violates (b). Thus $\gamma(p)=p$, or equivalently, $\gamma \in \Gamma_{p}$.

Proposition 3.3. Suppose that one of (a) and (b) in Proposition 3.2 is satisfied. Let $(D, \varphi)$ be a $\Gamma$-circularized domain. Then $p \in D$ is a pole of $\varphi$ if $\Gamma_{p}$ is nontrivial. 
Proof. Note that $D$ is noncompact and simply connected. Let $q$ be the pole of $\varphi$ in $D$. By the remark following Proposition 3.1 we know $\Gamma_{q}=\Gamma_{D}$. On the other hand $\Gamma_{p} \subset \Gamma_{D}$ by Lemma 3.1. Hence we have $\left\{\operatorname{id}_{R}\right\} \neq \Gamma_{p} \subset \Gamma_{D}=$ $\Gamma_{q}$. Since any nontrivial conformal automorphism of $D$ has at most one fixed point in $D$, it follows that $p=q$.

THEOREM 3.1. Let $R^{*}$ denote the set of points of $R$ which are fixed by no nontrivial elements of $\Gamma$. Let $D$ be a $\Gamma$-circularizable domain on $R$. If $D \subset R^{*}$, then $\gamma(D) \cap D=\emptyset$ for all nontrivial $\gamma \in \Gamma$.

Proof. If $R^{*}$ is conformally equivalent to $\hat{\mathbf{C}}$, then $\Gamma$ is trivial and there is nothing to prove. Otherwise $D$ is noncompact by Proposition 2.2 (ii') so that there exists $p \in D$ such that $\Gamma_{D}=\Gamma_{p}$ by Proposition 3.1. Since $D \subset R^{*}$, we know that $\Gamma_{D}=\Gamma_{p}=\left\{\mathrm{id}_{R}\right\}$. Now the theorem is an immediate consequence of Proposition 3.2.

Proposition 3.4. Assume that no elements of $\Gamma$ other than $\mathrm{id}_{R}$ have a fixed point on $R$. Then a domain $D$ on $R$ is $\Gamma$-circularizable if and only if $\pi(D)$ is circularizable, where $\pi$ is the natural projection of $R$ onto $R / \Gamma$. If this is the case, $\pi$ induces a bijection between the $\Gamma$-circularizers of $D$ and the circularizers of $\pi(D)$.

Proof. Suppose first that $(D, \varphi)$ is a $\Gamma$-circularized domain. Because of the $\Gamma$-invariance $\varphi$ is projected to a meromorphic quadratic differential $\psi$ on $\pi(R)$. By Theorem 3.1 we know that $\left.\pi\right|_{D}: D \rightarrow \pi(D)$ is a holomorphic bijection, which implies that $(\pi(D), \psi)$ is a circularized domain on $\pi(R)$.

Assume conversely that $(\pi(D), \psi)$ is a circularized domain on $\pi(R)$. Then the pullback $\varphi$ of $\psi$ via $\pi$ is a $\Gamma$-invariant meromorphic quadratic differential on $R$. Since $\pi: R \rightarrow \pi(R)$ is a smooth unlimited covering and $\pi(D)$ is simply connected by Proposition 2.1, it follows from the monodromy theorem that $\left.\pi\right|_{D}: D \rightarrow \pi(D)$ is a holomorphic bijection. Hence $(D, \varphi)$ is a $\Gamma$-circularized domain on $R$.

Remark. Let $\Gamma$ be an arbitrary group of automorphisms of $R$ acting properly discontinuously on $R$. If $D$ is a $\Gamma$-circularizable domain on $R$ with $D \subset R^{*}$, then $\pi(D)$ is a circularizable domain on $\pi(R)$.

\section{The boundary of a circularizable domain}

In this section we investigate geometric properties of boundaries of circularizable domains. The simplest cases have been investigated in Corollary 2.1 and Proposition 2.5. General circularizable domains have a fairly regular boundary. To describe the regularity we recall some definitions from algebraic geometry. By a geometric simplicial complex we mean a collection $K$ of affine simplices in $\mathbf{R}^{\infty}$ such that 
(a) if $\sigma \in K$, then any face of $\sigma$ is in $K$, and

(b) if $\sigma, \tau \in K$, then $\sigma \cap \tau$ is a face of both $\sigma$ and $\tau$, or is empty

(cf. [1, Definition IV.21.2]). Note that $K$ may be an infinite set. The dimension of $K$ is, by definition, the supremum of the dimensions of simplices in $K$. Set $|K|=\bigcup_{\sigma \in K} \sigma$. If for any $p \in|K|$ there is a neighborhood $U$ of $p$ in $\mathbf{R}^{\infty}$ such that $U$ meets only finitely many simplices in $K$, then we say that $K$ is locally finite. A topological space $X$ is said to be a polyhedron if there exists a homeomorphism of $|K|$ onto $X$ for some geometric simplicial complex $K$. If the dimension of $K$ is $n$, then $X$ is called an $n$-polyhedron. It is called locally finite if $K$ is.

We are particularly interested in 1-polyhedra, which will be called branched polygons. An end of a branched polygon $X$ is, by definition, a point of $X$ whose corresponding point on $|K|$ constitutes a 0 -simplex in $K$ which is included in exactly one 1 -simplex of $K$.

THEOREM 4.1. The boundary of a circularizable domain is a locally finite branched polygon if it contains more than one point.

For the proof we first study the behavior of circularizers on the relative boundary $\partial D$. $q \in \partial D$

Proposition 4.1. Suppose that $(D, \varphi)$ is a circularized domain. Then for

(i) $\operatorname{ord}_{q} \varphi \geqq-2$, and

(ii) if $\operatorname{ord}_{q} \varphi=0$, then the horizontal trajectory of $\varphi$ passing through $q$ lies entirely on $\partial D$.

Moreover, $\varphi$ has a double pole on $\partial D$ if and only if $R \cong \hat{\mathbf{C}}$ and $D \cong \mathbf{C}$.

Proof. By [5, Theorems 7.2 and 7.4] for any $q \in R$ with $\operatorname{ord}_{q} \varphi \leqq-2$ either

(a) there is a neighborhood $U$ of $q$ such that every horizontal trajectory ray of $\varphi$ which enters into $U$ tends to $q$, or

(b) there is a simply connected neighborhood $U$ of $q$ such that $U \backslash\{q\}$ is swept out by closed horizontal trajectories of $\varphi$.

If $\operatorname{ord}_{q} \varphi<-2$, then (b) never occurs.

Now, assume that $q \in \partial D$. If $\operatorname{ord}_{q} \varphi \leqq-2$, then we can find a neighborhood $U$ of $q$ as in (a) or (b). The intersection $U \cap D$ contains a noncritical point $q^{\prime}$ of $\varphi$. Since $\varphi$ is a circularizer of $D$, the horizontal trajectory $\tau^{\prime}$ passing through $q^{\prime}$ is closed. This shows that (a) does not occur. Hence $\operatorname{ord}_{q} \varphi=-2$, and $\tau^{\prime}$ stays in $U \cap D$. If $q^{\prime \prime} \in U \backslash\{q\}$ does not belong to $D$, then the closed horizontal trajectory passing through $q^{\prime \prime}$ cannot meet $D$. Since $D$ is connected, it follows that $q$ is an isolated point of $\partial D$. Hence $R \cong \hat{\mathbf{C}}$ and $D \cong \mathbf{C}$ by Corollary 2.1 (ii).

Next, let $q \in \partial D$ and $\operatorname{ord}_{q} \varphi=0$. Then there is a local coordinate $\zeta$ about $q$ with $\zeta(q)=0$ such that $\varphi$ has the representation $\varphi=d \zeta^{2}$ (cf. [5, Theorem 5.1]). The horizontal trajectory $\tau$ passing through $q$ does not meet $D$. Since $q$ is on the boundary of $D$, there is a sequence $\left\{q_{n}\right\}$ in $D$ for which $q_{n} \rightarrow q$ as $n \rightarrow \infty$. The 
horizontal trajectories $\tau_{n}$ containing $q_{n}$ lie entirely in $D$. The metric $\sqrt{|\varphi|}$ induces a distance function on $R$, which defines the same topology on $R$. Observe that for any $p \in \tau$ there exists a point $p_{n} \in \tau_{n}$ such that the $\varphi$-distance between $p$ and $p_{n}$ is at most the $\varphi$-distance between $q$ and $q_{n}$. Hence $p$ is in the closure of $D$. This proves that $\tau$ is on the boundary of $\partial D$.

Proof of Theorem 4.1. By Corollary 2.1 and Proposition 4.1 the boundary $\partial D$ has no isolated points and consists of horizontal trajectories and critical points of a circularizer $\varphi$ of $D$. If $\partial D$ itself is a closed horizontal trajectory of $\varphi$, then we have nothing to do. Otherwise, each of the horizontal trajectories is mapped onto an open segment on $\mathbf{C}$ by the mapping $p \mapsto \int^{p} \sqrt{\varphi}$. The Euclidean length of the segment is identical with the $\varphi$-length of the trajectory. The total sum of the $\varphi$-lengths of the trajectories does not exceed the $\varphi$-length of a closed horizontal trajectory of $\varphi$ in $D$. Therefore, $\partial D$ includes at most countably many horizontal trajectories.

If $q \in \partial D$, then $m:=\operatorname{ord}_{q} \varphi \geqq-1$ by Proposition 4.1. We can thus find a local coordinate $\zeta$ about $q$ with $\zeta(q)=0$ such that $\varphi$ has the representation

$$
\varphi=\left(\frac{m+2}{2}\right)^{2} \zeta^{m} d \zeta^{2}
$$

(cf. [5, Theorem 6.2]). Set $U_{\rho}=\left\{q^{\prime} \in R|| \zeta\left(q^{\prime}\right) \mid<\rho\right\}$ for $\rho>0$ and consider the curves

$$
\beta_{k}:(0, \rho) \ni t \mapsto \zeta^{-1}\left(t e^{(2 k+1) \pi i /(m+2)}\right), \quad k=0,1, \ldots, m+1,
$$

on $U_{\rho}$. Then $\beta_{k}$ is a vertical arc of $\varphi$. If $\beta_{k}^{-1}\left(U_{\rho} \cap D\right)$ is nonempty, then it is an open interval of the form $(s, \rho)$, where $0 \leqq s<\rho$, because no vertical arc of $\varphi$ can be a cross-cut of $D$. Replacing $\rho$ with a smaller one if necessary, we may assume that for each $k$ the set $\beta_{k}^{-1}\left(U_{\rho} \cap D\right)$ is either $(0, \rho)$ or empty. Note that if $\beta_{k}(t) \in D$, then the maximal horizontal arc in $U_{\rho}$ of $\varphi$ that passes through the point $\beta_{k}(t)$ stays entirely in $D$. Consequently, each sector

$$
\left\{q^{\prime} \in U_{\rho} \backslash\{q\} \mid \frac{2 k}{m+2}<\arg \zeta\left(q^{\prime}\right)<\frac{2(k+1)}{m+2}\right\}
$$

is either included in $D$ or disjoint from $D$, from which we conclude that $(\partial D \backslash\{q\}) \cap U_{\rho}$ consists of finitely many horizontal arcs of $\varphi$ emanating from $q$. It is now easy to see that $\partial D$ is a locally finite branched polygon.

We see from the above proof that the following proposition holds:

Proposition 4.2. Let $(D, \varphi)$ be a circularized domain on $R$ with ${ }^{\#} \partial D \geqq 2$. Then $\partial D$ is a branched polygon without ends if and only if $\varphi$ has no poles on $\partial D$.

Remark. The boundary of a circularizable domain on $R$ consists of piecewise analytic arcs unless it reduces to a point. 
The following proposition refines [4, Theorem 4.1] on boundaries of hyperbolically maximal domains.

COROllary 4.1. The boundary of a hyperbolically maximal domain for $\Gamma$ is a locally finite branched polygon without ends.

Proof. Let $D$ be hyperbolically maximal for $\Gamma$. Then $D \cong$ D. By $[4$, Proposition 3.1] a $\Gamma$-circularizer of $D$ has no poles on $\partial D$. Thus the corollary follows from Corollary 2.1 and Proposition 4.2.

Proposition 4.3. Let $D$ be a circularizable domain on $R$ with $\# \partial D \geqq 2$. Let $Q$ be a prime end of $D$ with impression in $R$. Then the interior angle of $D$ at $Q$ is equal to $2 \pi / m$ for some $m \in \mathbf{N}$.

The proof is exactly the same as that of [4, Proposition 4.2], and may be omitted.

\section{A characterization of hyperbolically maximal domains}

By Proposition 2.3, if $D$ is hyperbolically maximal for $\Gamma$, then it is $\Gamma$ circularizable. From the following theorem we know when the converse is true. For $E \subset R$ we define $\Gamma(E)=\bigcup_{\gamma \in \Gamma} \gamma(E)$. We set $\Gamma(p)=\Gamma(\{p\})$ for $p \in R$.

THEOREM 5.1. Suppose that $R^{*} / \Gamma \nsubseteq \mathbf{C}, \hat{\mathbf{C}}$. Then a $\Gamma$-circularizable domain $D$ on $R$ is hyperbolically maximal for $\Gamma$ if and only if

(i) $D \subset R^{*}$,

(ii) the area of $R \backslash \Gamma(D)$ vanishes, and

(iii) $\partial D$ is a branched polygon without ends.

Proof. The necessity of conditions (i)-(iii) follows from Corollary 4.1 and [4, Proposition 3.1]. To prove the sufficiency let $\varphi$ be a $\Gamma$-circularizer of $D$ and take a pole $p$ of $\varphi$ in $D$. Proposition 3.4 implies that $\pi(D)$ is a circularizable domain on $\pi\left(R^{*}\right)$ and that $\varphi$ is projected to a circularizer $\psi$ of $\pi(D)$. Since $\pi(D)$ is hyperbolic by the assumption that $R^{*} / \Gamma \not \mathbf{C}, \hat{\mathbf{C}}$, we know that $\pi(p)$ is the only pole of $\psi$ in $\pi(D)$ (cf. Proposition 2.2 (ii) and (ii')).

Set $E^{*}=R^{*} \backslash \Gamma(D)$. We claim that $\psi$ has no poles on $\pi\left(E^{*}\right)=\pi\left(R^{*}\right) \backslash \pi(D)$. Since $\pi\left(E^{*}\right)$ is of zero area by assumption (ii), it is exactly the boundary of $\pi(D)$. It thus follows from Theorem 4.1 that $\pi\left(E^{*}\right)$ is a locally finite branched polygon. Since $\pi$ is locally homeomorphic on $R^{*}$, we conclude that for any $q \in E^{*}$ there exists a neighborhood $U$ of $q$ for which $\left(E^{*} \backslash\{q\}\right) \cap U$ is a disjoint union of finitely many Jordan arcs with one end tending to $q$. Hence $q$ is on the boundary of a component of $U \backslash E^{*}$. Since this component is included in a $\Gamma$ image $\gamma(D)$ of $D$ and $E^{*}$ never meets $\Gamma(D)$, we infer that $q$ is on the boundary of $\gamma(D)$. This proves that $\pi\left(E^{*}\right)=\pi(\partial D) \cap \pi\left(R^{*}\right)$. By assumption (iii) the 
branched polygon $\pi\left(E^{*}\right)$ has no ends. Consequently, $\psi$ is holomorphic on $\pi\left(E^{*}\right)$ by Proposition 4.2.

We have shown that $\psi$ is holomorphic throughout on $\pi\left(R^{*}\right) \backslash\{\pi(p)\}$. In view of $\left[4\right.$, Proposition 3.1] we see that $D$, as a subdomain of $R^{*}$, is hyperbolically maximal for $\Gamma$. It then follows from [4, Proposition 2.1] that $D$, as a subdomain of $R$, is hyperbolically maximal for $\Gamma$, too.

Example 5.1. Fix an integer $k \geqq 2$ and let $G_{k}$ be the rotation group generated by $g_{k}: z \mapsto e^{2 \pi i / k} z$ acting on the unit disk $\mathbf{D}$. Then

$$
D_{k}:=\left\{z \in \mathbf{D}|| \arg z \mid<\frac{\pi}{k}\right\}
$$

is a $G_{k}$-circularizable domain. To show this, take a real $z_{0}$ in $D_{k}$ and let $w=f(z)$ be the conformal mapping of $\{z \in \mathbf{D} \mid 0<\arg z<\pi / k\}$ onto $\{w \in \mathbf{D} \mid$ $0<\arg w<\pi\}$ with $f(0)=-1, f\left(z_{0}\right)=0$ and $f(1)=1$. We use the Schwarz reflection principle to extend $f$ to a meromorphic function on $\{z \in \mathbf{D} \mid-\pi / k<$ $\arg z<3 \pi / k\}$. Because $f(\bar{z})=\overline{f(z)}$ and $f\left(g_{k}(\bar{z})\right)=1 / \overline{f(z)}$ hold for $z \in D_{k}$, we have $f\left(g_{k}(z)\right)=1 / f(z)$. Thus the function

$$
h:=\frac{1}{2}\left(f+\frac{1}{f}\right)
$$

satisfies $h \circ g_{k}=h$ on $D_{k}$, which enables us to extend $h$ to a single-valued meromorphic function on the punctured disk $\mathbf{D} \backslash\{0\}$. Since $f: D_{k} \rightarrow \mathbf{D}$ is conformal, 0 is a removable singularity of $h$. We note that $h$ is a meromorphic $G_{k^{-}}$ automorphic function on $\mathbf{D}$ with $h \not \equiv 1$. The $G_{k}$-invariant meromorphic differential

$$
\varphi:=\frac{h^{\prime}(z)^{2}}{1-h(z)^{2}} d z^{2}
$$

on $\mathbf{D}$ is a circularizer of $D_{k}$ since

$$
\varphi=\frac{\left\{\frac{f^{\prime}(z)}{2}\left(1-\frac{1}{f(z)^{2}}\right)\right\}^{2}}{1-\frac{1}{4}\left(f(z)+\frac{1}{f(z)}\right)^{2}} d z^{2}=-\frac{d w^{2}}{w^{2}}
$$

on $D_{k}$. By Theorem 5.1 the domain $D_{k}$ is hyperbolically maximal for $G_{k}$.

Remark. A hyperbolically maximal domain can be maximal simultaneously in two or more classes $\mathscr{D}_{p}^{\Gamma}(R)$. In fact in the above example the domain $D_{k}$ is maximal in $\mathscr{D}_{p}^{G_{k}}(\mathbf{D})$ for any real and positive $p \in \mathbf{D}$.

Example 5.2. For a positive integer $k$ let $\hat{G}_{k}$ be the dihedral group generated by the rotations $z \mapsto e^{2 \pi i / k} z$ and $z \mapsto z^{-1}$ of $\hat{\mathbf{C}}$. Then $\hat{\mathbf{C}}$ is a $\hat{\boldsymbol{G}}_{k^{-}}$ 
circularizable domain. In fact the $\hat{G}_{k}$-invariant meromorphic quadratic differential $-z^{-2} d z^{2}$ is a circularizer of $\hat{\mathbf{C}}$. Needless to say, $\hat{\mathbf{C}}$ is also a $G$-circularizable domain for any subgroup $G$ of $\hat{G}_{k}$.

We see from Example 5.2 that the converse of Proposition 2.4 is also valid. We thus have the following

Proposition 2.4'. There exists a compact $\Gamma$-circularizable domain on $R$ if and only if $R$ is conformally equivalent to $\hat{\mathbf{C}}$ and $\Gamma$ is either cyclic or dihedral.

Proof. We have only to show the "if part". Assume that $R \cong \hat{\mathbf{C}}$ and that $\Gamma$ is either cyclic or dihedral. Let $f$ be a conformal mapping of $R$ onto $\hat{\mathbf{C}}$. Then $f \circ \Gamma \circ f^{-1}$ is a (finite) cyclic or dihedral group of Möbius transformations. By normalizing $f$ so that $f \circ \Gamma \circ f^{-1}$ is a subgroup of $\hat{G}_{k}$ in Example 5.2 for some $k$ (see [2, Proof of Theorem 2.13.5]) we see that $R$ is $\Gamma$-circularizable.

\title{
REFERENCES
}

[ 1 ] G. E. Bredon, Topology and Geometry, Springer-Verlag, Berlin-Heidelberg-New York, 1993.

[2] G. A. Jones and D. Singerman, Complex Functions, an algebraic and geometric viewpoint, Cambridge Univ. Press, Cambridge, 1987.

[ 3 ] M. Masumoto and M. Shiba, Intrinsic disks on a Riemann surface, Bull. London Math. Soc. 27 (1995), 371-379.

[ 4 ] M. Masumoto ANd M. Shiba, Hyperbolically maximal domains and fundamental domains for a discontinuous group of conformal automorphisms of a Riemann surface, Q. J. Math. 53 (2002), 337-346.

[ 5] K. Strebel, Quadratic Differentials, Springer-Verlag, Berlin-Heidelberg-New York, 1984.

\author{
Department of Mathematics \\ YAMAGUCHI UNIVERSITY \\ YAMAGUCHI 753-8512 \\ JAPAN \\ e-mail: masumoto@yamaguchi-u.ac.jp \\ ApPliEd Mathematics \\ Graduate School of EngineEring \\ HiRoshima UNIVERSITY \\ Higashi-HiRoshima 739-8527 \\ JAPAN \\ e-mail: shiba@amath.hiroshima-u.ac.jp
}

